\title{
Parabolic Equation Modeling in Horizontally Inhomogeneous Environments
}

\author{
Amalia E. Barrios
}

\begin{abstract}
A parabolic equation (PE) model has been developed for use in tropospheric radiowave propagation. A simple technique to model range-dependent environments has been implemented. Results from the model are compared with experimental data at $170,520,3240,3300$, and $9875 \mathrm{MHz}$ in measured range-dependent environments. The experimental data are taken from two separate experiments performed during 1947 and 1948. Measurements were made on overwater paths from Guadalupe Island to San Diego, $\mathrm{CA}$, in one experiment, and the other was located in the South Island of New Zealand, also known as the Canterbury Project. The results are presented as one-way propagation factor in decibels versus height. The technique used here to model range-dependent environments is shown to give a reasonably good estimate of the environment between measurements, leading to excellent agreement between the predicted fields and observed radio data.
\end{abstract}

\section{INTRODUCTION}

$\mathrm{T}$ HERE have been many papers in recent years on the development of the split-step parabolic equation method as applied to electromagnetic wave propagation [1]-[5]. The parabolic equation method was originally developed by Fock in 1946 [6], but it wasn't until 1973 that a practical solution for complicated refractive environments was developed by Hardin and Tappert, called the split-step Fourier method [7]. This method was originally applied to model acoustic propagation, but the radar community has since used the split-step algorithm to model propagation in the troposphere.

The importance of the split-step parabolic equation method is that not only does it provide an exact solution to field equations (within the operator approximation) for a homogeneous atmosphere, but it can also predict (with relatively small errors) field strengths for vertically and laterally inhomogeneous environments in a relatively short time. The more conventional mode-conversion methods can also be used [4], but the time involved in obtaining numerical results is generally too long to be of any practical consideration.

Over the sea the troposphere usually exhibits horizontal homogeneity over long distances. It has been found that the assumption of a horizontally stratified troposphere leads to valid operational propagation assessments $86 \%$ of the time [8]. However, the environment can change drastically at air/mass boundaries associated with wave cy-

Manuscript received July 26, 1991; revised February 21, 1992.

The author is with the Research and Development, Test and Evaluation Division, Naval Command, Control and Ocean Surveillance Center, Code 543, San Diego, CA 92152

IEEE Log Number 9200789. clones and land/ocean interfaces [9]. The importance of being able to adequately model an environment that varies with range will become apparent as case studies are presented between experimental data and the predicted fields.

A parabolic equation model for the personal computer (PEPC) has been developed by Professor F. Tappert of the University of Miami in conjunction with the Naval Ocean Systems Center (NOSC) at San Diego during 1989 and 1990. Comparisons between predicted PE fields and measured radio and meteorological profiles on overwater paths from Guadalupe Island to San Diego in 1947 and 1948 [10] are presented. Some comparisons were also made against experimental data taken from the Canterbury Project [11].

A theoretical description of the derivation of the splitstep parabolic equation will not be given here, but rather, a brief description of the algorithm and its implementation will be given in Section II. The emphasis of this paper is on the validation of the PE model based on comparisons with experimental data, and also the use of a simple technique by which range-dependent environments can be adequately modeled. For a detailed description of the theory the reader is referred to [2], [6], [12], and [13]. Range-independent and range-dependent comparisons are made in Sections III and IV, respectively.

\section{SPLIT-STEP PE AlgorithM}

In order to solve for the electromagnetic field at any point above the earth's surface, one must solve Maxwell's equations subject to boundary conditions, making certain assumptions about the medium. The troposphere is assumed to vary in range and height only, making the field equations independent of azimuth. The earth's surface will be considered smooth and perfectly conducting. The scalar Helmholtz wave equation is derived from Maxwell's equations through a change of variable recommended by Fock [6], and following the methods given in [2], [12], and [13] the scalar component of the field at range $x+\Delta x$ is now approximated as

$\begin{aligned} u(x+\Delta x, z)=e^{i k_{o}(\Delta x / 2)\left(m^{2}(x, z)-1\right) \mathscr{F}}-1 & \cdot\left[e^{-i(\Delta x / 2)\left(p^{2} / k_{o}\right) \mathscr{F}}[u(x, z)]\right]\end{aligned}$

where the Fourier transform is defined as

$$
\begin{array}{r}
U(x, p)=\mathscr{F}[u(x, z)]=\int_{-\infty}^{\infty} u(x, z) e^{-i p z} d z \\
u(x, z)=^{-1}[U(x, p)]=\frac{1}{2 \pi} \int_{-\infty}^{\infty} U(x, p) e^{i p z} d p .
\end{array}
$$


The transform variable $p$ is defined by $k_{o} \sin \vartheta . \vartheta$ is the propagation angle above the horizontal and $k_{o}$ is the free-space wavenumber. While (2a) and (2b) define the continuous Fourier transform, what is actually implemented in the model is the discrete Fourier transform. This necessarily bandlimits the transform variable $p$ to a predefined value corresponding to a maximum propagation angle, in which case the limits of integration in (2b) $(-\infty<p<\infty)$ would be $-p_{\max }<p<p_{\max }$ for the discrete transform. Similarly, integration in height, or the $z$ parameter in (2a), would be limited to a maximum height, following Nyquist's criteria. The modified index of refraction, $m(x, z)$, takes into account the earth's curvature and is defined by $m(x, z)=n(x, z)+z / a$, where $a$ is the earth's radius and $n$ is the index of refraction. Qualitatively, (1) represents the field at range $x$ undergoing free-space propagation over the range interval $\Delta x$ (bracketed term), which is then attenuated (or modulated) by the actual medium within that same range interval.

The derivation resulting in (1) uses the standard PE (SPE) approximation, leading to the SPE propagator (exponential term in brackets in (1)). The SPE approximation was originally derived by Tappert. Another widely used approximation, resulting in a modified form of the propagator in (1), is the wide-angle PE (WAPE). The WAPE propagator was found to give more accurate numerical predictions at higher angles than the SPE propagator [14] However, only small propagation angles (within $1^{\circ}$ ) are subject to trapping in the troposphere, and within the small angular range usually dealt with in tropospheric propagation, Dockery states he found no significant difference between the two propagators [13]. Therefore, only the SPE propagator is used in the model.

The field given by (1) is exact, within the SPE approximation, for range-independent environments. When the modified refractive index $m(x, z)$ is range dependent, a somewhat complicated error term is present. This error term depends on the range step size $\Delta x$, the frequency, and the refractive index gradients. Therefore, one can make the errors associated with a range dependent refractive index in (1) small by taking a sufficiently small step size and assuming $m(x, z)$ varies slowly with height and range.

A mixed radix fast Fourier transform algorithm developed by Bergland [15], combined with algorithms for sine and cosine transforms by Cooley, Lewis, and Welsh [16] are used. The maximum propagation angle above the horizontal and the maximum calculation height are made to depend on the frequency, thereby limiting the program to low altitude, small-angle propagation effects at high frequencies. To keep reflections (inherent in the use of the discrete Fourier transform) from entering the physical portion of the calculation domain, a cosine-taper (Tukey) window [17] is used to attenuate the field smoothly at large heights and large propagation angles.

The source is assumed to be horizontally polarized. The field at any point above the earth can be found using image theory and the appropriate boundary conditions.
For a perfectly conducting surface and horizontal polarization, the field must vanish at the surface: $u(x, 0)=0$. The next step is to find an initial field $[u(0, z)]$ for which (1) can then be used to "march" the field out in range. One first begins with noting that the field at range zero is essentially the antenna aperture distribution, and that the far-field antenna pattern and its aperture distribution are a Fourier transform pair:

$$
u(0, z) \stackrel{\mathscr{F}}{\leftrightarrow} U(0, p)=f(p) .
$$

The antenna pattern $f(p)$ is assumed to be symmetric about $p=0$ (0 elevation angle). Introducing a source height and elevation angle is easily done by using the Fourier transform shift properties, however, a zero elevation angle was used in all results presented in this paper. A truncated sinc aperture distribution (essentially an omnidirectional antenna $[f(p)=1]$ ) was used for the antenna pattern.

A troposcatter model is included and was taken from Yeh [18] and Rice et al. [19]. For a full description of the model see [20]. To keep the error term [associated with a nonconstant $m$ in (1)] small, we must keep $\Delta x$ small. $\Delta x$ is made to depend on the wavelength, thereby forcing the program to take small range steps at large frequencies where it is needed.

For environmental inputs, the program accepts height versus refractivity profiles at specified ranges. Modified refractivity ( $M$-unit) is used and is defined by

$$
M(z)=\left[n-1+\frac{z}{a}\right] \times 10^{6}
$$

More will be said about the method of interpolation for range-dependent environments in Section IV.

\section{HoMOGENEOUS ENVIRONMENTS}

A radio-meteorological investigation was conducted jointly by the Departments of Scientific and Industrial Research of the United Kingdom and New Zealand under the name of the Canterbury Project in Canterbury Province in the South Island of New Zealand [11]. Transmitters were located in aircraft that flew a sawtooth pattern from over $100 \mathrm{~km}$ off shore inward toward the receiving ground stations located at Wakanui Beach. Data were taken from the Canterbury Project for the afternoon of August 5, 1947. This case was chosen because the environment varied little with range. Fig. 1 shows a strong surface duct present from $20 \mathrm{~km}$ off shore out to $100 \mathrm{~km}$. Slant paths were used for the model so as to represent different "legs" of the aircraft flight paths. Figs. 2 and 3 show two comparisons at 9875 and $3240 \mathrm{MHz}$, respectively. Both figures display propagation factor versus height for one "leg" from 120 to $140 \mathrm{~km}$, in which the aircraft descended from $300 \mathrm{~m}$ (at $120 \mathrm{~km}$ ) to near the ocean surface (at $140 \mathrm{~km}$ ). Propagation factor is defined as the ratio of the magnitude of the field at a point in space to the magnitude of the field at the same point under freespace conditions [21]. A height gain curve for a standard atmosphere is shown as a reference. The model compares well with the observed data. 


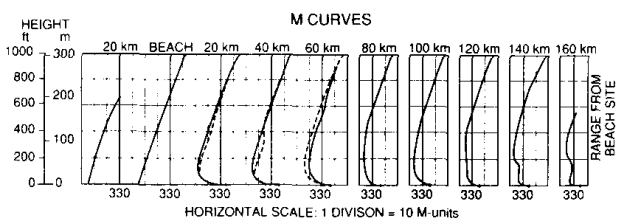

Fig. 1. Canterbury Project meteorological measurements for August 5, 1947. Dashed curves represent measurements taken during 1415-1515 hours, solid curves represent measurements taken during 1300-1415.

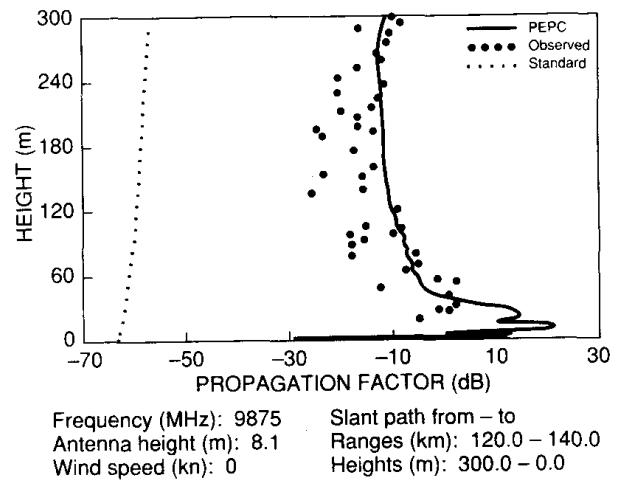

Fig. 2. Comparison between PEPC and measured radio data at 9875 $\mathrm{MHz}$ from the Canterbury project for August 5, 1947.

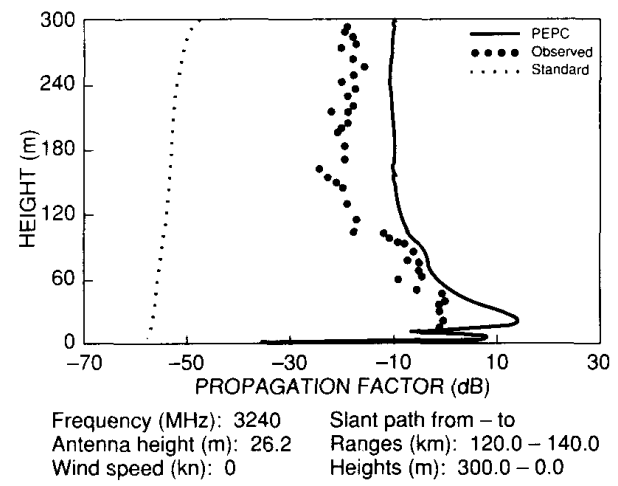

Fig. 3. Comparison between PEPC and measured radio data at 3240 MHz from the Canterbury project for August 5, 1947.

\section{INHOMOGENEOUS ENVIRONMENTS}

At every range step the split-step algorithm requires a refractivity profile as a function of height. For a range-dependent environment, a height versus $M$-unit profile, along with its corresponding range is specified. Since the range step $\Delta x$ is made to depend on the frequency, $\Delta x$ can be as much as $900 \mathrm{~m}$ for low frequencies and as little as 270 $\mathrm{m}$ for very high frequencies. For a region of interest, say $100 \mathrm{~km}$, entering a height-refractivity profile at every range step is quite impractical. In most cases, only a few meteorological measurements are made over a distance of 100 or $200 \mathrm{~km}$.
To determine the most likely occurring environment between two that are measured, a linear interpolation in range is made for each height/refractivity pair. This method of interpolation provides for logical and smooth transitioning of the various features between specified profiles. Interpolating horizontally on refractivity alone does not give an adequate representation of the environment between two known profile measurements.

As an extreme case, three profiles and their ranges were specified as inputs to PEPC. The first profile consists of a single, standard atmosphere gradient. The second profile, at $100 \mathrm{~km}$, is a $300 \mathrm{~m}$ surface-based duct with a trapping layer thickness of $50 \mathrm{~m}$ and a gradient of -990 $\mathrm{M} / \mathrm{km}$. The third profile, at $200 \mathrm{~km}$, is a $600 \mathrm{~m}$ surfacebased duct with a trapping layer thickness of $100 \mathrm{~m}$, also with a gradient of $-990 \mathrm{M} / \mathrm{km}$. One would logically assume, based on the profiles at 100 and $200 \mathrm{~km}$ that the trapping layer is linearly increasing, not only in height but in thickness as well, as shown in Fig. 4(a). The shaded area represents the trapping layer. Notice the strength, or gradient, of the trapping layer remains the same. Fig. 4(b) shows the same three profiles with the shaded area representing the trapping layer height and thickness based on refractivity-only interpolation. The darker shaded areas show where the gradient is strongest. When interpolating only on refractivity the trapping layer will remain at the same height and thickness, however, its gradient will change. Notice at some range interval between 100 and $200 \mathrm{~km}$ there will appear to be two trapping layers. The resulting numerical predictions based on these two methods of interpolation will look quite different, as shown in the coverage diagrams, Figs. 5(a) and 5(b), respectively.

The very severe gradient given above does not violate the conditions for the validity of the split-step PE method. A gradient of $-990 \mathrm{M} / \mathrm{km}$ corresponds to $|\partial n / \partial z|$ of about $1.15 \times 10^{-6} \mathrm{~m}^{-1}$ and $|\partial n / \partial x|$ on the order of $10^{-9}$ $\mathrm{m}^{-1}$. Both conditions 24 and 25 of [13] are easily satisfied even in the presence of such a strong and sharply varying ducting condition. Interpolation on both height and refractivity will be referred to as "parametric" interpolation in the remainder of the paper and is implemented in the following comparisons.

Radio and meteorological data were measured concurrently in overwater paths from Guadalupe Island to San Diego during 1947 and 1948 [10]. In this experiment receivers were located on the ground and the transmitting and meteorological equipment was located in the aircraft. The aircraft flew a sawtooth pattern and took radio measurements on both ascending and descending paths, but only made meteorological measurements on the ascending paths. The slant profiles are represented vertically at ranges corresponding to the optimum coupling height. The optimum coupling height is the height at which the base of the inversion layer is measured, and where placement of an antenna at that height will result in maximum angular trapping. Figs. 6(a) and (b) shows the meteorological data records for the two days of March 12, 1948 and November 13, 1947, respectively. The data are plotted in 


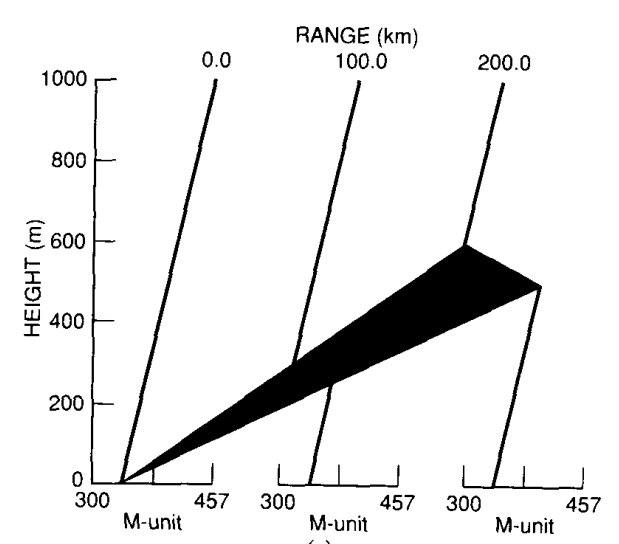

(a)

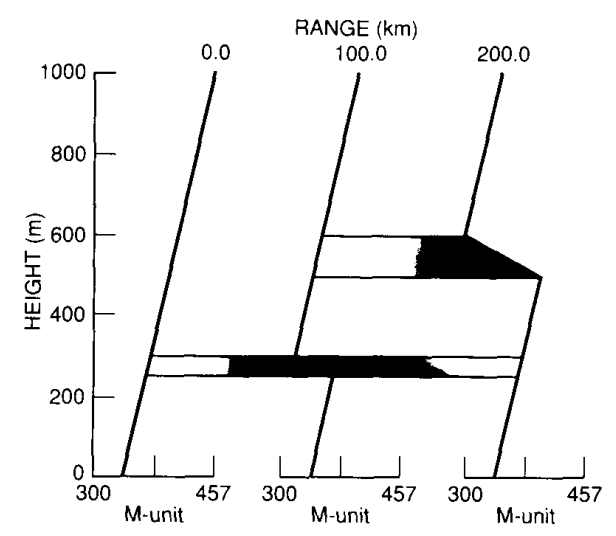

(b)

Fig. 4. (a) Range-dependent environment varying from standard atmosphere at $0 \mathrm{~km}$ to $600 \mathrm{~m}$ surface-based duct at $200 \mathrm{~km}$. Shaded area represents trapping layer location based on linear interpolation on height and refractivity. (b) Range-dependent environment as in (a) with shaded area representing trapping layer location based on linear interpolation on refractivity only. Density of shading represents duct strength.

feet versus nautical miles with refractivity plotted in $B$ units, defined as

$$
B=(n-1) 10^{6}+0.039 \mathrm{~h}
$$

for $h$ in meters.

The measurements of March 12, 1948 show a slowly increasing trapping layer (shaded area) from 40 to 200 $\mathrm{nm}$. Five linear segments were taken of each profile. The environment between San Diego and the first measured profile was assumed to be homogeneous, since meteorological data was not available at the shore site. Measurements made on November 13, 1947 show a trapping layer increasing rather steeply. On this day soundings were made at the shore site and were used for the environment at range 0 . Radio measurements were made at 170,520 , and $3300 \mathrm{MHz}$ for both days. The receiver height is located at $100 \mathrm{ft}$ in all of the following Guadalupe Island results for PEPC.

Figs. 7(a)-7(c) shows comparisons between PEPC and March 12, 1948 observed data for frequencies at 170,520, and $3300 \mathrm{MHz}$, respectively. The slant path used is la-

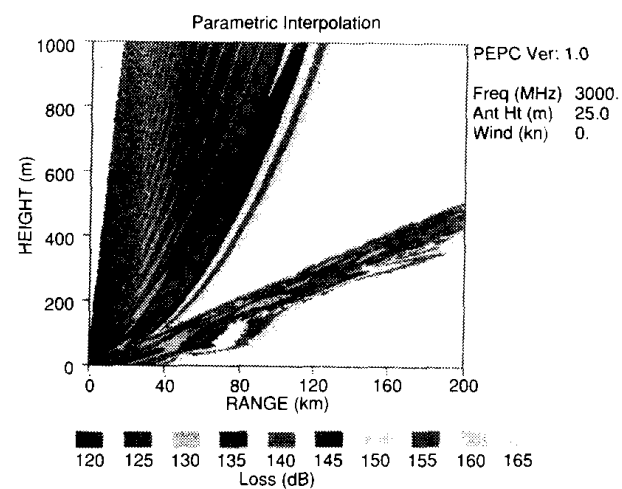

(a)

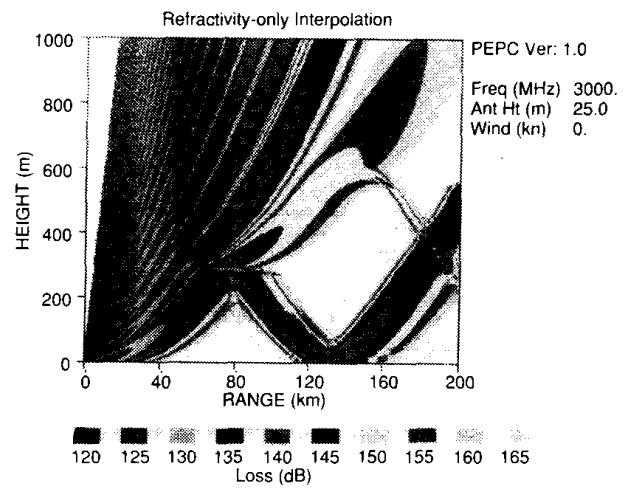

(b)

Fig. 5. (a) PEPC coverage diagram for environment of Fig. 4(a) using parametric interpolation. (b) PEPC coverage diagram for environment of Fig. 4(b) using refractivity-only interpolation.

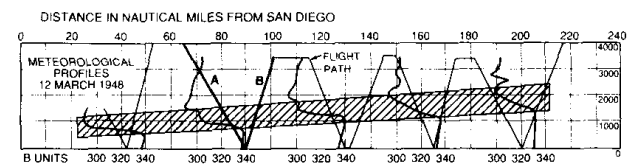

(a)

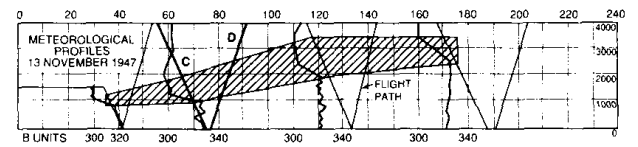

(b)

Fig. 6. (a) Guadalupe Island meteorological measurements for March 12, 1948. (b) Guadalupe Island meteorological measurements for November 13, 1947.

beled $\mathrm{A}$ in Fig. 6(a). In all three cases the model agrees very well. In most of the measurements made at 3300 $\mathrm{MHz}$, the experimenters observed a great deal of signal fluctuation at certain height intervals. This data was digitized at both the maximum and minimum signal levels, appearing as two curves in Fig. 7(c). Notice in this region the predicted field level also fluctuates.

Figs. $8(\mathrm{a})-8(\mathrm{c})$ is the 170,520 , and $3300 \mathrm{MHz}$ comparisons, respectively, for November 13,1947 . The slant paths 


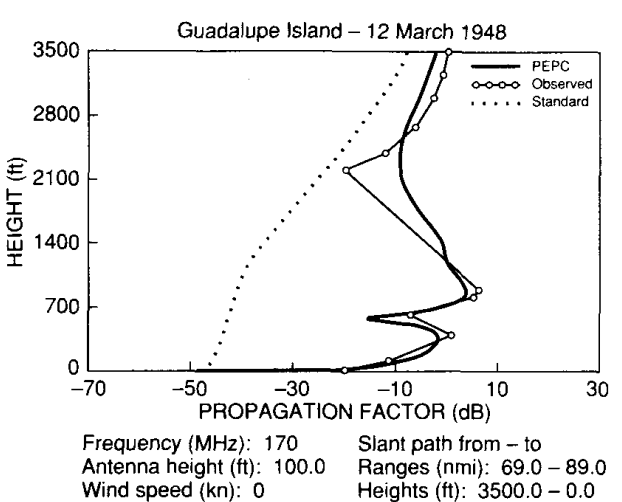

(a)

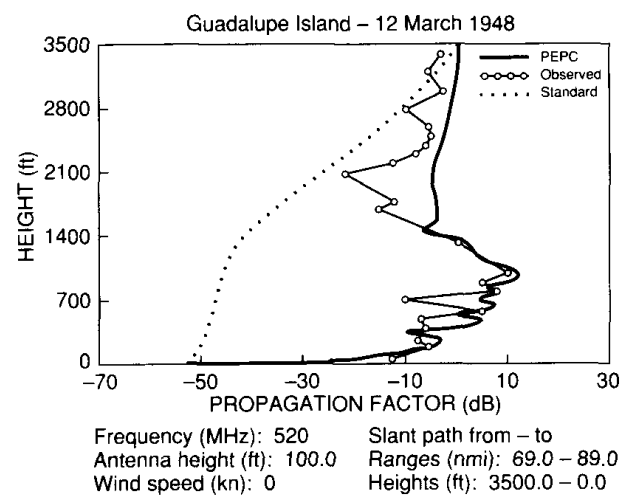

(b)

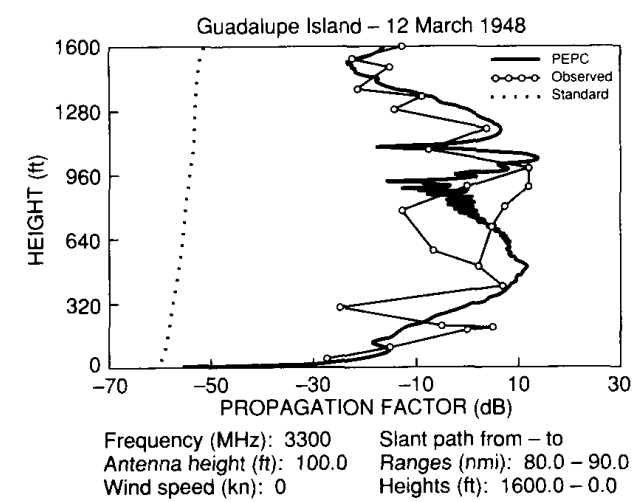

(c)

Fig. 7. (a) Comparison between PEPC and measured radio data at 170 $\mathrm{MHz}$ for March 12, 1948 along slant path labeled A in Fig. 6(a). (b) Comparison between PEPC and measured radio data at $520 \mathrm{MHz}$ for March 12, 1948. (c) Comparison between PEPC and measured radio data at $3300 \mathrm{MHz}$ for March 12, 1948.

for Figs. 8(a) and (b)-(c) are labeled C and D, respectively, in Fig. 6(b). Again, good agreement is shown between the model and measured data.

Typically, the environment does not vary greatly in range, as mentioned in Section I. In some cases, if enough environmental measurements are made separated by 10 or $20 \mathrm{~km}$, "refractivity-only" interpolation may be adequate, since over a small range step the change in height is very slight. However, as is usually the case, the number

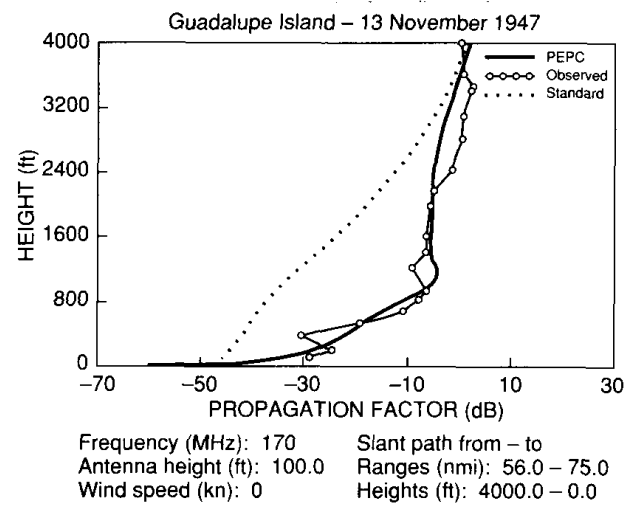

(a)

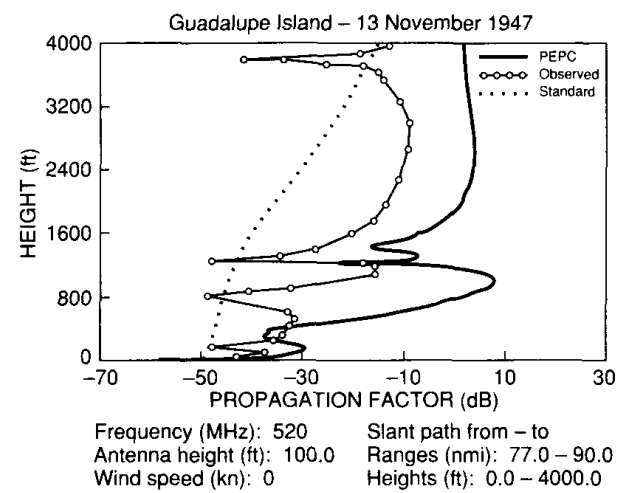

(b)

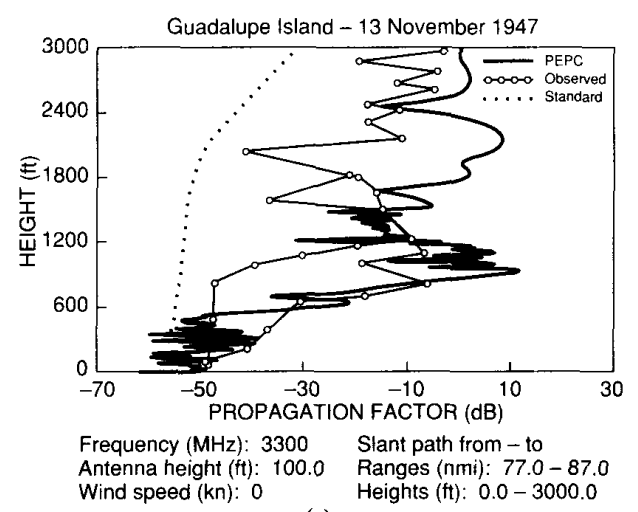

(c)

Fig 8 . (a) Comparison between PEPC and measured radio data at 170 MHz for November 13, 1947 along slant path labeled C in Fig. 6(b). (b) Comparison between PEPC and measured radio data at $520 \mathrm{MHz}$ for November 13, 1947 along slant path labeled D in Fig. 6(b). (c) Comparison between PEPC and measured radio data at $3300 \mathrm{MHz}$ for November 13, 1947 along slant path labeled D in Fig. 6(b).

of soundings available are few and separated by large distances.

To demonstrate the advantage in using parametric interpolation rather than interpolating in refractivity only, the second and third profiles of Fig. 6(a), at 80 and 120 nmi, respectively, were omitted for March 12, 1948. The field must now be computed based on the best assumption of how the environment is changing within the two measured profiles at 40 and $160 \mathrm{nmi}$. Comparisons were made, 
along slant path B in Fig. 6(a), against measured data using "refractivity-only" interpolation (dotted curve) and parametric interpolation (solid curve), shown in Fig. 9. While the predicted field using "refractivity-only" interpolation does not differ greatly from the measured field, the predicted field compares better (and the lobe structure at the lower heights were more tractable) using parametric interpolation. A better illustration of this is shown using the meteorological data for November 13,1947. Here, the second profile of Fig. 6(b) was omitted, and slant path $\mathrm{C}$ was used for the comparison shown in Fig. 10. On this day the trapping layer varied much more sharply, thereby "demanding" a proper estimate of the changes in the trapping layer with range and height. The solid curve, using parametric interpolation in Fig. 10, gives a much better match to the measured field than does the refractivity-only (dotted) curve. Compare the solid curve in Fig. 10 with the solid curve in Fig. 8(a) (in which all meteorological profiles were included). Clearly, parametric interpolation is the better method.

The meteorological measurements made during the Canterbury Project are shown as height versus $M$-unit curves in Figs. 11(a) and 11(b) for June 19 and July 11, 1947 , respectively. For June 19, 1947, two comparisons are shown at $9875 \mathrm{MHz}$ with an $8.1 \mathrm{~m}$ antenna height [Fig. $12(\mathrm{a})]$ and at $3240 \mathrm{MHz}$ with the antenna height at $26.2 \mathrm{~m}$ [Fig. 12(b)]. Fig. 13 shows a comparison at $9875 \mathrm{MHz}$ for July 11, 1947. There is excellent agreement in all three cases.

\section{CONCLUSION}

The parabolic equation model, PEPC, has been shown to predict, with reasonably good accuracy, field strengths for range-dependent and range-independent environments. Although the model is simple in that it uses smooth surface and infinite conductivity, for the cases presented here, it agreed very well with experimental data.

The simple technique of interpolating both height and refractivity, called parametric interpolation, to model horizontally varying environments was shown to give excellent agreement between the predicted field strengths and the observed data, even when vertical profile measurements were separated by large distances. While PEPC internally performs parametric interpolation on the vertical profiles, the user must specify, upon input, how the environment is changing. Specifically, which features or vertical inflections on which to interpolate. The environment given for March 12, 1948 was very well defined in that every vertical profile could be taken to consist of five major linear segments, and the change with range of each segment could be easily seen. However, this is not always the case, and the difficulty with specifying how the environment changes should not be underestimated.

\section{ACKNOWLEDGMENT}

The author wishes to thank Professor F. Tappert, Mr. R. Paulus, and Mr. H. Hitney for helpful discussions.

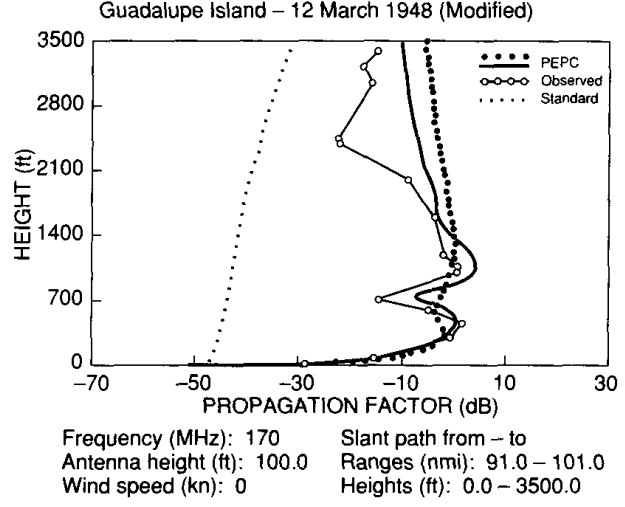

Fig. 9. Comparison between predicted and measured field levels based on parametric interpolation (solid curve) and "refractivity-only" interpolation (dotted curve) between profiles at 40 and $160 \mathrm{nmi}$ along slant path labeled B in Fig. 6(a).

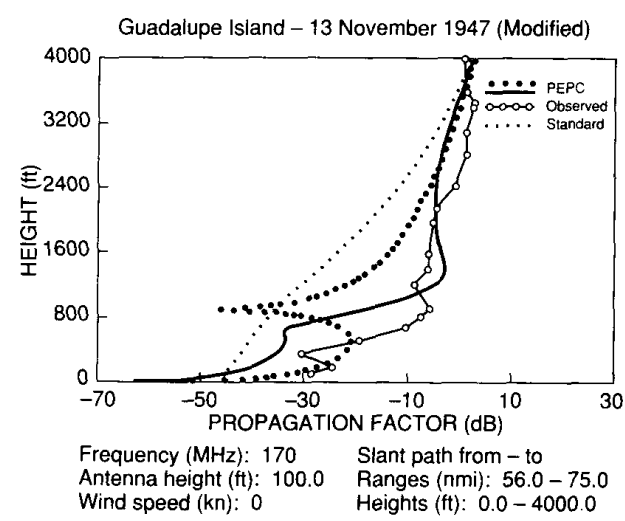

Fig. 10. Comparison between predicted and measured field levels based on parametric interpolation (solid curve) and "refractivity-only" interpolation (dotted curve) between profiles at 40 and $120 \mathrm{nmi}$ along slant path labeled C in Fig. 6(b).

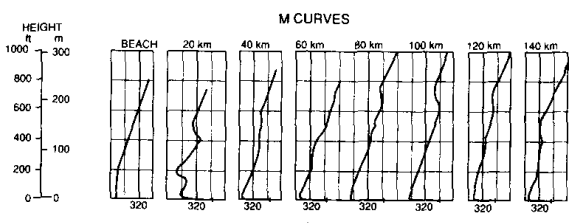

(a)

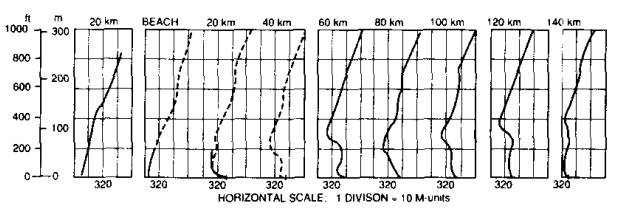

(b)

Fig. 11. (a) Canterbury Project meteorological measurements for June 19, 1947. (b) Canterbury Project meteorological measurements for July 11, 1947. Dashed curves represent measurements taken during 1130-1210 hours, solid curves represent measurements taken during 1030-1130 hours. 


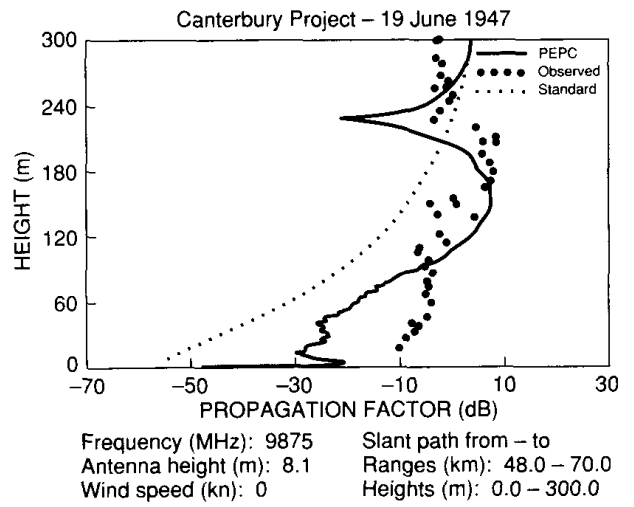

(a)

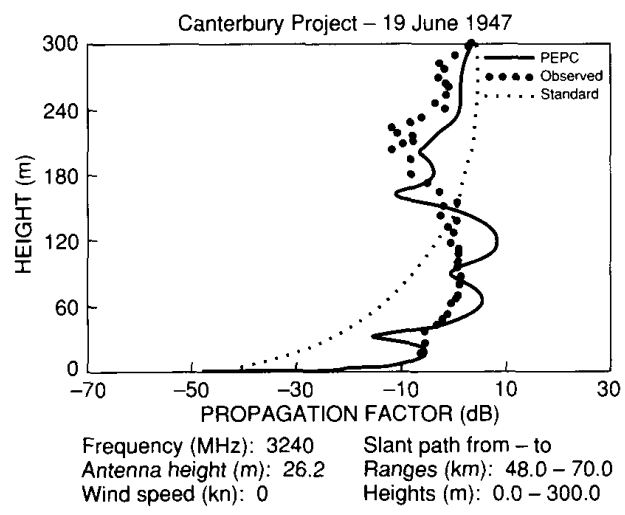

(b)

Fig. 12. (a) Comparison between PEPC and measured radio data at $9875 \mathrm{MHz}$ for June 19, 1947. (b) Comparison between PEPC and measured radio data at $3240 \mathrm{MHz}$ for June 19, 1947.

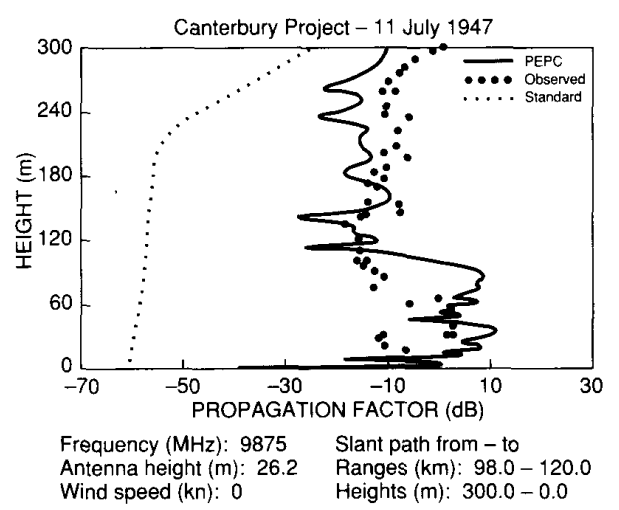

Fig. 13. Comparison between PEPC and measured radio data at 9875 MHz for July $11,1947$.

\section{REFERENCES}

[1] G. D. Dockery and G. C. Konstanzer, "Recent advances in prediction of tropospheric propagation using the parabolic equation," Johns Hopkins APL Tech. Dig., vol. 8, pp. 404-412, 1987.

[2] F. J. Ryan, "RPE: A parabolic equation radio assessment model," in AGARD Operational Decision Aids for Exploiting or Mitigating Electromagnetic Propagation Effects, 1989.

[3] K. H. Craig, "Propagation modeling in the troposphere: Parabolic equation method," Electron. Lett., vol. 24, pp. 1136-1139, 1989.
[4] G. D. Dockery, "Modeling electromagnetic wave propagation in the troposphere using the parabolic equation," IEEE Trans. Antennas Propagat., vol. 36, pp. 1464-1470, Oct. 1988.

[5] H. W. Ko, H. S. Burkom, J. P. Skura, and D. A. Roberts, "An analysis of EMPE code performance in a selection of laterally inhomogeneous atmospheric-duct environments," Johns Hopkins APL Tech. Dig., vol. 9, no. 2, pp. 89-100, 1988.

[6] V. A. Fock, Electromagnetic Diffraction and Propagation Problems. New York: Pergamon, 1965, ch. 11-14.

[7] R. H. Hardin and F. D. Tappert, "Application of the split-step Fourier method to the numerical solution of nonlinear and variable coefficient wave equations," SIAM Rev., vol. 15, p. 423, 1973.

[8] H. V. Hitney, J. H. Richter, R. A. Pappert, K. D. Anderson, and G. B. Baumgartner, Jr., "Tropospheric radio propagation assessment," Proc. IEEE, vol. 73, pp. 265-285, Feb. 1985.

[9] W. L. Patterson, "A Raytrce method for a laterally heterogeneous environment," NOSC TR, vol. 1180, July 1987.

[10] Symp. Tropospheric Wave Propagat., Naval Electron. Lab., San Diego, CA, Rep. 173, July 1949.

[11] Great Britain Dept. Scient. Indust. Res., Report of Factual Data from the Canterbury Project, Vols. I, II, III, 1951.

[12] F. D. Tappert, "The parabolic approximation method", in Wave Propagation and Underwater Acoustics (Lecture Notes in Physics), J. B. Keller and J. S. Papadakis, Eds. New York: Springer-Verlag, 1977, vol. 70, pp. 224-287.

[13] J. R. Kuttler and G. D. Dockery, "Theoretical description of the parabolic approximation/Fourier split-step method of representing electromagnetic propagation in the troposphere," Radio Sci., pp. 381-393, Mar.-Apr. 1991.

[14] M. D. Feit and J. A. Fleck, Jr., "Light propagation in graded-index fibers," Appl. Opt., vol. 17, pp. 3990-3998, 1978.

[15] G. D. Bergland, "A radix-eight fast Fourier transform subroutine for real-valued series," IEEE Trans. Audio Electro-Acoust., vol. AU-17, pp. 138-144, 1969.

[16] J. W. Cooley, P. A. W. Lewis, and P. D. Welsh, "The fast Fourier transform algorithm: Programming considerations in the calculation of sine, cosine, and laplace transforms," J. Sound Vib., vol. 12, pp. 315-337, 1970.

[17] F. J. Harris, "On the use of windows for harmonic analysis with the discrete Fourier transform," Proc. IEEE, vol. 66, pp. 51-83, Jan. 1978.

[18] L. P. Yeh, "Simple methods for designing troposcatter circuits," IRE Trans. Commun. Syst., vol. CS-8, pp. 193-198, 1960.

[19] P. L. Rice, A. G. Longley, K. A. Norton, and A. P. Barsis, "Transmission loss predictions for tropospheric communication circuits," U.S. Dept. of Commerce, Nat. Bureau of Stand., Technical Note 101, vols. 1 and 2, 1965.

[20] W. L. Patterson, C. P. Hattan, H. V. Hitney, R. A. Paulus, A. E. Barrios, G. E. Lindem, and K. D. Anderson, "Engineer's refractive effects prediction system (EREPS) revision 2.0," NOSC TD 1342, Feb. 1990.

[21] D. E. Kerr, Propagation of Short Radio Waves. New York: McGraw-Hill, 1951, ch. 2

[22] K. H. Craig and M. F. Levy, "Field strength forecasting with the parabolic equation: Wideband applications," presented at Sixth Int. Conf. Antennas and Propagat. (ICAP 89), Warwick, U.K

[23] N. W. Guinard, M. B. Laing, and K. W. Morin, "Ray tracing in rising and falling ducts," NRL Rep. 6253, June 22, 1965.

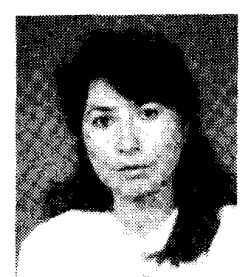

Amalia E. Barrios was born in Mendota, CA, in 1962. She received the B.S. degree in physics from California State University, Fresno, in 1983 and the M.S. degree in electrical engineering from the University of California, San Diego, in 1989.

In 1983 she joined the Research and Development, Test and Evaluation Division of the Naval Command, Control and Ocean Surveillance Center (formerly Naval Ocean Systems Center) in San Diego, CA. She is currently working on applying parabolic equation techniques to model tropospheric radiowave propagation over variable terrain. 